\title{
Role and Effective Therapeutic Target of Gut Microbiota in Heart Failure
}

\author{
Qiujin Jia, ${ }^{1}$ Hao Li, ${ }^{1}$ Huan Zhou, ${ }^{2}$ Xiaonan Zhang $\left(\mathbb{D},{ }^{1}\right.$ Ao Zhang, ${ }^{3}$ Yingyu Xie, ${ }^{4}$ Yanyang Li, ${ }^{5}$ \\ Shichao Lv $\mathbb{D}^{1}{ }^{1}$ and Junping Zhang $\mathbb{D}^{1}$ \\ ${ }^{1}$ First Teaching Hospital of Tianjin University of Traditional Chinese Medicine, 314 An Shan Xi Road, Nan Kai District, \\ Tianjin 300193, China \\ ${ }^{2}$ Affiliated Hospital of Nankai University, 4 Wei Shan Road, He Xi District, Tianjin 300222, China \\ ${ }^{3}$ Epidemiology, College of Global Public Health, New York University, 726 Broadway, New York, NY 10003, USA \\ ${ }^{4}$ Tianjin University of Traditional Chinese Medicine, 10 Po Yang Lake Road, Tuan Bo New Town West, Jing Hai District, \\ Tianjin 301617, China \\ ${ }^{5}$ Tianjin Medical University Cancer Institute and Hospital, Tianjin 300193, China
}

Correspondence should be addressed to Shichao Lv; 372272027@qq.com and Junping Zhang; tjzhtcm@163.com

Received 29 May 2019; Revised 16 September 2019; Accepted 19 September 2019; Published 16 November 2019

Academic Editor: Prasanth Puthanveetil

Copyright (C) 2019 Qiujin Jia et al. This is an open access article distributed under the Creative Commons Attribution License, which permits unrestricted use, distribution, and reproduction in any medium, provided the original work is properly cited.

\begin{abstract}
Although the mechanism of the occurrence and development of heart failure has been continuously explored in the past ten years, the mortality and readmission rate of heart failure is still very high. Modern studies have shown that gut microbiota is associated with a variety of cardiovascular diseases, among which the study of gut microbiota and heart failure attracts particular attention. Therefore, understanding the role of gut microbiota in the occurrence and development of heart failure will help us further understand the pathogenesis of heart failure and provide new ideas for its treatment. This paper introduced intestinal flora and its metabolites, summarized the changes of intestinal flora in patients with heart failure, clarified that intestinal barrier damage and bacterial translocation induced inflammation and immune response aggravated heart failure, and altered intestinal microflora affected various metabolic pathways including trimethylamine/TMAO, SCFA, and Bile acid pathway leads to heart failure. At the same time, regulating intestinal microflora through diet, probiotics, antibiotics, fecal transplantation and microbial enzyme inhibitors has grown up to be a potential treatment for many metabolic disorders.
\end{abstract}

\section{Introduction}

Heart failure is a severe and terminal stage of many cardiovascular diseases and is an important part of the global prevention and treatment of chronic cardiovascular diseases. Epidemiological data show that the prevalence of heart failure in adults is $1 \%$ to $2 \%$ and increases to more than $10 \%$ of people over the age of $70[1,2]$. With the ageing of the population, the incidence of chronic diseases such as coronary heart disease, hypertension, diabetes, obesity is on the rise, and the improvement of medical level, the survival time of patients with heart disease is prolonged, resulting in a continuous increase in the prevalence of heart failure. Heart failure is a difficult clinical syndrome caused by a variety of causes of abnormal changes in cardiac structure and function, resulting in ventricular systolic and/or diastolic function disorders [3]. Currently, heart failure is considered as a chronic, spontaneous and progressive disease, and the activation of the neuroendocrine system leads to pathological myocardial remodelling, which is the crucial factor in the occurrence and development of heart failure [4]. In the field of modern medical treatment, many drugs are being used, including beta-blockers, angiotensin-converting enzyme inhibitors and angiotensin receptor blockers (ARB), aldosterone antagonists, and combination of $\mathrm{ARB} /$ neprilysin blockers, ivabradine [5]. However, current treatments target only a fraction of the putative pathophysiological pathways, the overall prognosis of heart failure remains poor, readmission rates and mortality rates remain high, and even in the PARADIGM study, the 2-year mortality rate in the trial group was as high as $20 \%$ [6]. In addition, patients with 
heart failure are under a low quality of life, and long-term medication imposes a heavy financial burden on patients. Therefore, prevention of heart failure, timely diagnosis and early treatment are key to successful mortality reduction and prognosis. Gut microbiota is a unique ecosystem, and it functions as an endocrine organ, produces a plethora of metabolism dependent and metabolism-independent signals that play regulatory roles in cardiovascular disease development in the host [7]. More and more studies have shown that gut microbiota is closely related to the occurrence and development of heart failure, so microbiota is expected to become an essential target for intervention of heart failure.

\section{Gut Microbiota and Its Metabolites}

Intestinal micro-ecosystem is composed of gastrointestinal tract lumen, epithelial cell secretion, gut microbiota and substances entering the intestinal tract. Gut microbiota is the most important active ingredient in intestinal microecosystem [8]. The human body harbors 10-100 trillion microbes, mainly bacteria in our gut, which greatly outnumber our human cells [9]. The gut microbiota in the human body is mainly composed of Bacteroides, Firmicutes, Actinobacteria, Proteobacteria and Verrucomicrobia. Among them, Firmicutes and Bacteroides are dominant, accounting for more than $90 \%$ of the total intestinal microflora, and the remaining bacteria are less than $1 \%$ of the total gut microbiota $[10,11]$. Owing to differences in host genes and external environmental factors (e.g., use of antibiotics, diet structure, lifestyle), the proportion of this flora is different in different individuals or different organs of the same individual $[12,13]$. Flora can not only participate in the food digestion and nutrient uptake, providing energy for the host but also secrete metabolites, which can be viewed as hormone-like factors by dedicated receptor systems in the human host [14].

At present, gut microbiota interacts with the host through metabolism-independent pathways, such as lipopolysaccharide (LPS) and peptidoglycan, which are bacterial cell wall products, and metabolite-driven pathways, such as short-chain fatty acid (SCFA), trimethylamine (TMA)/trimethylamine $\mathrm{N}$-oxide (TMAO) and bile acid (BA) [7]. Intestinal flora produces SCFAs by decomposing dietary fiber, mainly including acetic acid, propionic acid and butyric acid. The proportion of them in the colon is about $60: 25: 15$ [15]. SCFAs can provide energy for intestinal epithelial cells and can also be involved in metabolic, immune and inflammatory responses as signaling molecules [16]. TMAO is only a little molecule compound. It is mainly transformed from choline, phosphatidylcholine and L-carnitine-rich foods (red meat, poultry, fish and eggs) by intestinal microorganisms to TMA, which are produced under the action of hepatic and flavin monooxygenase (FMO) [17]. BA is a vital part of bile. Primary bile acid changes into secondary bile acid through microbiota, and the composition changes of the bile acid pool can also affect the distribution of gut flora [18]. BAs can facilitate the absorption of dietary fat, fat-soluble molecules and cholesterol [19]. Besides, the gut microbiota is involved in the formation and regulation of the intestinal mucosal barrier [20], controlling nutrient intake, storage and metabolism [21], assisting the maturation of immune tissues, and preventing the growth of pathogenic microorganisms in the body [22]. Under physiological conditions, the intestinal flora is located in a state of balance. Once the balance is broken, pathogenic microorganisms thrive, leading to intestinal related diseases, such as inflammatory bowel disease, obesity, allergic diseases, diabetes, autism, colorectal cancer and cardiovascular diseases. In recent years, intestinal flora and cardiovascular diseases including coronary heart disease, hypertension, and heart failure have received sustained attention, and more and more evidence indicates that there is a close relationship between intestinal flora and heart failure [23-25].

\section{Gut Microbiota Dysbiosis in Heart Failure}

Exogenous factors such as diet, exposure to bacterial infections or taking drugs can reduce the diversity of intestinal flora; endogenous factors such as acute humoral imbalance, chronic intestinal congestion or ischemia-hypoxia, acid-base imbalance, weakened gastrointestinal motility, and nutritional deficiency can potentially change intestinal flora [26]. With the development of heart failure, the community characteristics of bacteria have changed. Studies have shown that the intestinal flora abundance of chronic heart failure patients decreased, and the number of pathogenic bacteria increased significantly with the progress of the disease, including Campylobacter, Shigella, Salmonella, Yersinia enterocolitica and Candida species [27]. According to Luedde et al., heart failure cases showed a significant decrease in Coriobacteriaceae, Erysipelotrichaceae and Ruminococcaceae at the family level and a significant decrease in Blautia, Collinsella, unclassifified (uncl.) Erysipelotrichaceae and uncl. Ruminococcaceae at the genus level [28]. A 16SrDNA analysis based on 22 hospitalized patients with heart failure reported a reduction in SCFA producing bacteria such as Eubacterium rectale and Dorea longicatena [29]. In addition, another study also shows that the gut microbiota signature in chronic heart failure is characterized by large compositional shifts with low bacterial richness and depletion of bacteria with butyrate-producing potential [30]. Butyrate exerts local anti-inflammatory effects in the gut mucosa and stimulates regulatory T-cells, also in the periphery [31]. Cui et al. observed that microbial genes for LPS biosynthesis and TMAO generation were up-regulated and genes for butyrate acetoacetate coenzyme A transferases (the key enzyme for the generation of butyrate) was down-regulated in chronic heart failure [25]. In conclusion, the intestinal flora of patients with heart failure changed, beneficial bacteria decreased, and pathogenic bacteria increased. The occurrence and development of heart failure may be linked to the decrease of SCFA-producing bacteria and the increase of TMAO-producing bacteria, which may become a new target for the treatment of heart failure.

\section{The Role of Gut Microbiota in the Development of Heart Failure}

4.1. Gut Barrier Dysfunction and Inflammation. Recently, more and more studies have confirmed that the intestinal 
tract plays an important role in the pathogenesis of heart failure, which is often referred to as the "gut hypothesis of heart failure". The gut hypothesis implies that decreased cardiac output and redistribution of systemic circulation can lead to a decrease in intestinal perfusion and mucosal ischemia, which creates hypoxia and a hypercapnia status [32]. Subsequently, a decrease in intestinal mucosal $\mathrm{PH}$ and diminished activity of passive carrier-mediated transport occurs [32, 33], leading to a "leaky gut," which describes increased gut permeability as well as intestinal barrier dysfunction. This disruption in intestinal barrier function, in turn, can lead to increased gut permeability, increased bacterial translocation and increased circulating endotoxins that can contribute to the underlying inflammation seen in patients with heart failure [32]. Sandek et al. proved that patients with chronic heart failure had increased thickness of the intestinal wall, intestinal permeability, and intestinal insufficiency [33]. A study also found that patients with moderate to severe congestive heart failure had increased intestinal permeability that observed a $78 \%$ increase via a sugar cellobiose test compared to the healthy controls, with a correlation between high right atrial pressure and increased intestinal permeability [27]. Another study by Sandek et al. showed that a higher concentration of juxta mucosal anaerobic bacteria in the sigmoid colon in patients was correlated with a higher systemic concentration of antiLPS IgA antibodies and above a certain threshold, the more bacteria there are in the biofilm, the higher the LPS antibodies [34]. More definitive investigations have identified increased concentrations of endotoxin, specifically LPS, in edematous patients with heart failure [35]. Besides, during acute heart failure, a higher LPS concentration was found in the hepatic vein compared with the left ventricle, suggesting that bacteria migrated from the intestine to the systemic circulation [36]. The circulating endotoxins generated by bacteria refer to the main structural components of bacteria, including LPS, peptidoglycans, and so on. LPS and peptidoglycans interact with host mucosal surface cells through pattern recognition receptors such as toll-like receptors (TLRs) and nucleotide oligomerization domain-containing receptors (NODs) to recognize microbe-associated molecular patterns (MAMPs), stimulate and direct host immune responses [7, 37]. It is currently believed that low levels of gut-derived bacteria can appear in the circulation, leading to chronic low-grade inflammation, known as "metabolic endotoxemia", which has been found in many chronic metabolic diseases such as obesity, type 2 diabetes and atherosclerosis [38]. Of course, this chronic low-grade inflammation can undoubtedly accelerate the development of heart failure. LPS-induced TLR4 activation induces the release of inflammatory cytokines like tumour necrosis factor- $\alpha$ (TNF- $\alpha$ ), interleukin (IL)-1 and IL-6 [39]. However, these inflammatory mediators are associated with cardiac apoptosis, hypertrophy, and fibrosis [33]. Additionally, LPS itself can continue to promote deterioration of the mucosal barrier function. When LPS was up-regulated, the expression of ZO-1(Zonulaoccludens-1) and Occludin decreased $[40,41]$, and intestinal permeability increased. Therefore, the assessment of future intestinal barrier function may help us gain a better understanding of the occurrence and development of heart failure.

4.2. Intestinal Mucosal Immunity. Recent studies have shown that the immune activation mechanism marked by elevated inflammatory cytokines plays a vital role in the development of chronic heart failure. The interaction between gut microbiota and mucosal immunity is related to the occurrence of heart failure. Th17 cells are a subtype of $\mathrm{CD}_{4}^{+}$helper T cells, which is critical to the body to resist bacterial and fungal infections. Th17 cells play a role in the development of autoimmune diseases by secreting inflammatory factors such as IL-17, IL-22, IL-21 and recruiting neutrophils. Studies have demonstrated that sectional filamentous bacteria can promote the differentiation of Th17 cells in mice. The mechanism may be that sectional filamentous bacteria can induce the expression of serum amyloid A after colonisation into host epithelial cells, which can stimulate the secretion of IL- 6 and IL-23 by dendritic cells in the lamina propria of the intestine, thereby promoting the differentiation of Th17 cells [42]. Clinical studies showed that Th17 cells in Acute Viral Myocarditis patients were hyperfunction and helped $B$ cells to generate anti-myocardial antibodies [43]. Necessary studies have shown that IL-17 can promote myocardial inflammation and myocardial ischemia reperfusion injury $[44,45]$. These results suggest that gut microbiota can encourage the development of chronic heart failure by influencing intestinal mucosal immunity. Polysaccharide A secreted by Bacteroides fragilis can induce $\mathrm{CD}_{4}^{+} \mathrm{T}$ cells to transform into Foxp ${ }^{3+}$ Treg cells, and Foxp ${ }^{3+}$ Treg cells can secrete anti-inflammatory factor IL-10 to regulate intestinal mucosal immune tolerance [46]. Treg cells control the abnormal expression of $\mathrm{T}$ cell receptors and $\mathrm{CD}_{4}{ }^{+} \mathrm{T}$ cell proliferation, inhibit intestinal inflammation, and secrete inflammatory inhibitors TGF- $\beta$ and IL-10, which mediate intestinal mucosal immune stability [47]. In addition, the research found that Treg cells can reduce ventricular remodelling after infarction by reducing apoptosis of myocardial cells and myocardial fibrosis [48]. Therefore, it is expected to improve heart failure by mediating intestinal mucosal immunity, thus providing a novel target for the treatment of heart failure.

4.3. The Gut Microbiota Metabolite-TMAO. Compared with patients with no heart failure, TMAO levels were higher in patients with chronic heart failure and associated with NYHA (New York Heart Association) grades, ischaemic aetiology and adverse outcomes [49]. A meta-analysis of 19 prospective studies in 19256 subjects showed that elevated plasma TMAO levels were associated with an increased relative risk of major adverse cardiovascular events, and the increase in relative risk did not change with BMI, diabetes, history of cardiovascular disease, renal dysfunction and other variables [50]. The TMAO level was significantly correlated with unfavourable outcomes (mortality and re-hospitalisation) in 2234, new or worsening heart failure patients, but guideline-based drug therapy did not affect the level of TMAO [51]. Besides, the study has shown that TMAO levels are associated with high BNP (B-type natriuretic peptide) levels and late left ventricular diastolic 
dysfunction, and can predict significant clinical adverse reactions for five years [52].

We have mentioned that TMAO levels are raised in chronic heart failure patients, but the mechanism of their increase is multifactorial. Changes in the composition of intestinal bacteria have turned out to be the principal drivers of TMAO levels [53]. Of course, TMAO levels are likewise primarily affected by TMAO substrate intakes, such as choline and betaine. Moreover, chronic heart failure patients have impaired intestinal mucosal barrier and increased permeability, which makes TMAO easier to enter the bloodstream through the intestinal mucosal barrier, leading to elevated levels. When mice with normal intestinal flora were fed a choline-rich diet, circulating TMAO levels increased, causing foam cell aggregation and promoting atherosclerotic plaque formation [17]. Mechanistic studies demonstrate that TMAO acts to potentiate platelet reactivity through alterations in stimulus-dependent calcium signaling [54]. Therefore, TMAO can increase atherosclerosis and thrombosis, which are entwined in the upstream aetiologies that assist in heart failure of ischaemic or non-ischaemic origin. Additionally, TMAO can induce cardiac hypertrophy and myocardial fibrosis in rats with aortic constriction, stimulate the increase of cardiac cell area and the expression of atrial natriuretic peptide and $\beta$-myosin heavy chain [55]. In addition, TMAO can activate NLRP3 (nucleotidebinding oligomerization domain-like receptor family pyrin domain-containing-3) inflammatory bodies to induce vascular inflammation through SIRT3-SOD2-mtROS (sirtuin-3-superoxide dismutase 2-mitochondrial reactive oxygen species) pathway [56], and also induce the expression of inflammatory genes in primary human aortic endothelial cells and vascular smooth muscle cells by activating nuclear factor(NF)-kB pathway [57]. TMAO can also up-regulate vascular cell adhesion molecule- 1 expression, promoted monocyte adherence, activated protein kinase $\mathrm{C}$ and NF-kB [58]. These results demonstrate that TMAO may encourage the development of chronic heart failure by accelerating endothelial dysfunction, including decreasing endothelial self-repair and activating the inflammatory response. Studies in animal models have shown that TMAO pathway can directly lead to confrontational myocardial remodelling and the development of heart failure phenotypes. In the experiment of heart failure rats induced by transverse aortic arch constriction model, TMAO can promote ventricular remodelling, decrease left ventricular pump function, myocardial interstitial cells and perivascular fibrosis [59]. In mice fed a high choline diet, severe adverse ventricular remodelling and fibrosis were observed to be significantly increased, and the profibrotic TGF- $\beta$-Smads pathway was activated [60]. Savi and colleagues [61] observed that it worsened cardiomyocyte contractility in the presence of TMAO in vitro. Most importantly, the nature of the TMAO receptor remains unknown. TMAO is considered to act as a small molecule (a protein chaperone mimetic) that alters the conformation of the protein [62]. Therefore, TMAO may affect signalling pathways not only through classical receptor-ligand interactions but also as allosteric modifiers. TMA is observed through the GPCR trace amine-associated receptor 5 (TAAR5). TAAR5 has a high affinity with TMA but does not recognise TMAO [63].
Therefore, there is further optimism about the discovery of receptor-mediated TMAO effects.

4.4. The Gut Microbiota Metabolite-SCFA. SCFA signal via G-protein-coupled-receptors(GPCRS) such as GPR41, GPR43, and GPR109A and are essential regulators of gut homeostasis and epithelial barrier maintenance [64, 65]. Accumulated evidence indicates that SCFAs play a role in mediating the host immune system. For example, butyric acid regulates gene expression by inhibiting histone deacetylase (HDAC), thus increasing the number of Treg cells and enhancing their functions, to achieve anti-inflammatory purpose [66]. In addition, SCFAs could modulate host blood pressure. Propionate increases blood pressure by inducing renin secretion by binding to Olfr78, an olfactory receptor expressed in the glomerular paracycles of the kidney. However, propionate can also lower blood pressure by guaranteeing to GPR41 [15]. Moreover, SCFAs play a gut barrier-protective role. Butyrate can promote the proliferation and differentiation of intestinal epithelial cells, repair damaged intestinal mucosa, maintain the integrity of the intestinal mucosa, and reduce inflammation caused by exogenous substances such as bacteria and their metabolites entering the blood circulation [67]. SCFAs can also promote post infarction cardiac repair through inducing infiltration of CX3CR1+ monocytes in the peri-infarct zone [68]. In conclusion, SCFA can inhibit the occurrence and development of inflammation through multiple mechanisms, which are expected to improve the occurrence and development of heart failure.

4.5. The Gut Microbiota Metabolite-BAs. BAs are currently recognized as signalling molecules, and emerging evidence suggests that BAs affect cardiovascular function. The understanding of bile acid physiology was greatly expanded by the discovery of bile acid-responsive receptors, such as the farnesoid X receptor (known as FXR) and G-protein coupled bile acid receptor 1 (also known as TGR5). According to a crosssectional study, an increased ratio of secondary to primary BAs in serum was found in patients with chronic heart failure, and this ratio was revealed to be associated with reduced overall survival in univariate analysis [69]. However, FXR can increase the imbalance of bile acid ratio, inhibit NF-kb, thereby reducing inflammation and improving myocardial function [70]. Bile acids, specifically TGR 5 agonists, induce cytoprotective changes in the heart and improve myocardial response to physiologic, inotropic, and hemodynamic stress in mice [71]. Therefore, TGR5 agonists and FXR may be novel targets for the treatment of heart failure in the future (Figure 1).

4.6. Gut Microbiota and Hepatic Health. Numerous physiological processes in the body are accomplished through two-way interaction between the intestine and the liver. Through the portal vein system, intestinal flora transports various metabolic or immune substances, bacterial components or products to the liver [72], and the liver can also affect the intestinal function by secreting bile or immune factors, making the intestine and liver closely linked, known as the intestinal-hepatic axis. When intestinal flora is misregulated, the portal vein can be used as the channel 


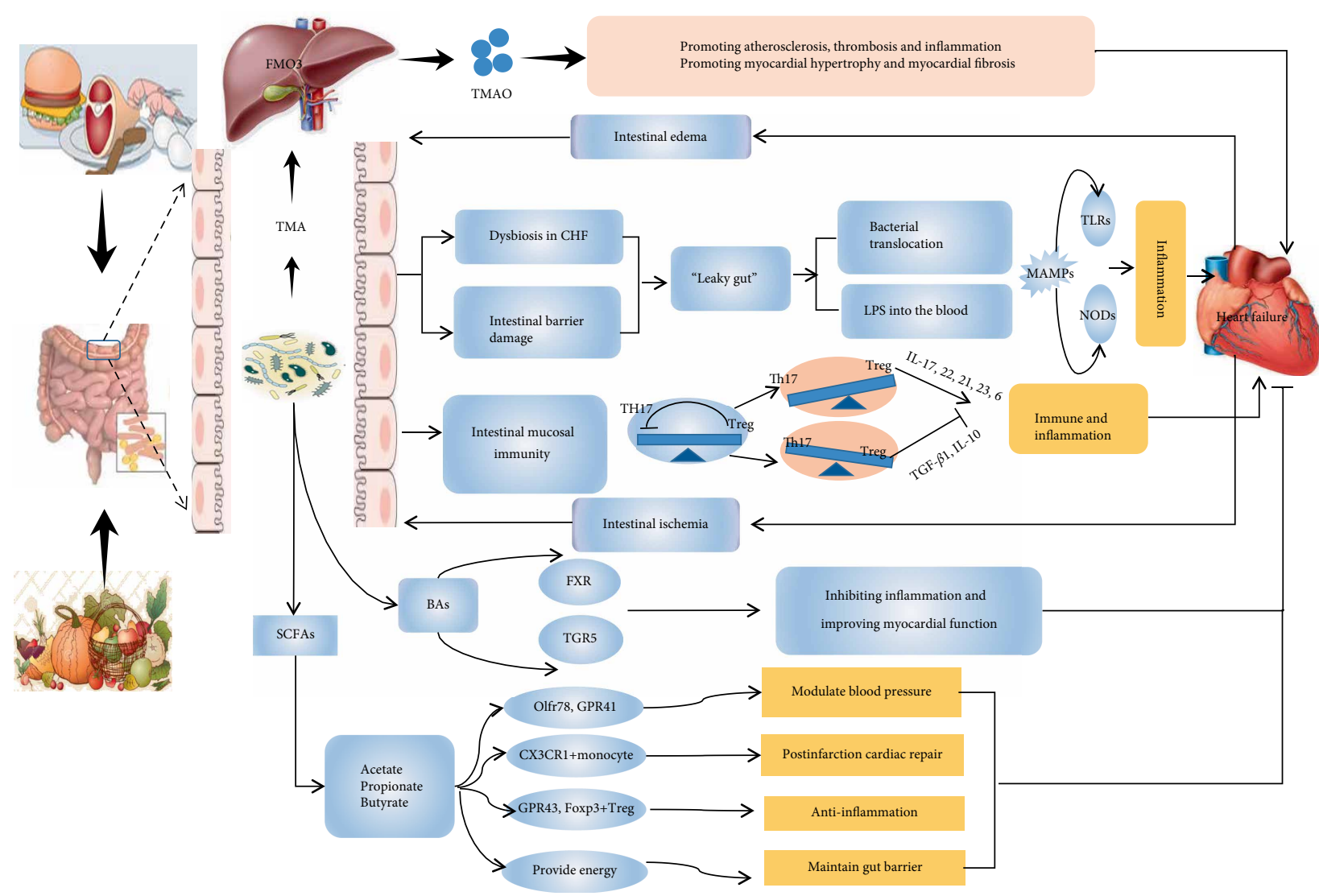

FIgURE 1: The role of gut microbiota in heart failure. TMA: Trimethylamine; TGF- $\beta$ : transforming growth factor- $\beta$; TMAO: trimethylamine N-oxide; IL: interleukin; SCFA: short-chain fatty acids; LPS: lipopolysaccharide; FMO3: flavin monooxygenase-3; Treg: regulatory T cells; Th17: helper T cells 17; GPR: G protein receptor; MAMPs: microbe-associated molecular patterns; NOD: nucleotide oligomerization domain; FXR: farnesoid $X$ receptor; TGR5: G-protein coupled bile acid receptor 1.

between the intestinal tract and liver, so that endotoxin and peptidoglycan from intestinal tract continue to enter the liver. Many liver cells express innate immune cell receptors, such as toll-like receptors, which respond to intestinal microbial products and activate the immune response of the liver, leading to liver injury [73]. Current research shows that heart failure is closely connected with the liver and can lead to liver damage when heart failure occurs. We have previously known that enterotoxin metabolites leak into the systemic circulation during heart failure. Since the liver is the first organ to come into contact with toxic intestinal molecules, the interaction between the intestine and the liver during heart failure has become an exciting new field of research [74].

4.7. Gut Microbiota and Renal Health. Intestinal flora is closely related to the kidney. It was found that there were significant differences in the types and quantity of intestinal flora between patients with end-stage renal disease and healthy people [75]. In patients with chronic kidney disease, dominant intestinal flora can usually induce local or systemic inflammation, damage the intestinal mucosal barrier function and cause an inflammatory response, and translocate LPS and intestinal bacterial components into systemic blood circulation by increasing Th17/Treg ratio [76]. Cardiovascular diseases are closely linked to kidney diseases. In cardiorenal syndrome, heart-kidney interaction usually results in accelerated deterioration of two organs [77]. In the intestine, urea is hydrolysed by microorganisms to form a large amount of ammonia, which is then converted to ammonium hydroxide. Ammonia and ammonium hydroxide can destroy the tight junction of the intestinal epithelium, leading to the destruction of intestinal epithelial barrier function, make intestinal bacteria DNA and endotoxin into the systemic circulation, leading to the systemic inflammatory response [78]. Also, gut microbiota ferments tryptophan and tyrosine in food to generate uremic toxin indole sulfate and p-cresol sulfate. They can activate downstream mitogen-activated protein kinase (MAPK) and NF-kB by activating apoptotic signal regulator enzyme (ASK) -1 , which mediates cardiac hypertrophy and cardiorenal fibrosis [79].

\subsection{Gut Microbiota and Normal Cardiovascular}

Health. Typically, the intestinal flora is in balance and protects the cardiovascular system through metabolites. For example, enterolactone is produced primarily by intestinal digestion of fibre-rich foods. A study shows that a high serum enterolactone level is associated with reduced cardiovascular diseases mortality [80]. Protocatechuic acid is one of the main metabolites of complex polyphenols such as anthocyanins and procyanidins that are normally found in high concentrations 
in vegetables and fruit [81]. The study concluded that protocatechuic acid has an anti-atherosclerotic effect by promoting reverse cholesterol transport [82].

\section{Gut Microbiota Interventions for Heart Failure}

At present, the improvement or reversal of intestinal microflora has become a hot spot for heart failure, which contains the dietary intervention, prebiotic and probiotic therapy, faecal microbiota transplantation, antibiotic intervention, TMAlyase inhibitors and so on.

5.1. Dietary Interventions. Diversity of gut microbiota was substantially correlated dietary habits, which confirmed the effect of long-term dietary patterns on gut microbiota. Studies have shown that adjusting diet for five days (short-term) can change the number and species of gut microbiota and produce corresponding changes to be adapted to dietary changes [83]. Dietary Approaches to Stop Hypertension (DASH) eating plan is a diet that is rich in fruit, vegetables, whole grains, and low-fat dairy foods. It includes meat, fish, poultry, nuts, and beans, and is bounded in sugar-sweetened foods and beverages, red meat, and added fats. The study has shown that the DASH diet can decrease the incidence of heart failure $[84,85]$. Compared with patients receiving conventional heart failure management guidelines, patients receiving the DASH diet had better 6-minute walking test performance, quality of life, and tended to increase arterial elasticity after a 3-month intervention [86]. The Mediterranean diet refers to the eating styles of vegetables, fruit, fish, cereals, beans and olive oil in the southern European countries of the Mediterranean coast. This diet has been proven to prevent cardiovascular disease and reduce mortality from cardiovascular disease [87]. Studies have shown an increase in urinary TMAO levels in patients who do not comply with the Mediterranean diet [88]. Also, a high-fibre diet can improve the growth of acetateproducing bacteria, reduce blood pressure, and inhibit cardiac hypertrophy and fibrosis [89].

5.2. Prebiotic and Probiotic Therapy. Probiotics mainly include bifidobacteria, yeasts, lactic acid bacteria, and so on. They can inhibit inflammation, protect and repair intestinal mucosal barrier, and improve intestinal function. A study in rats showed that probiotics (Lactobacillus rhamnosus GR-1) could significantly improve left ventricular hypertrophy and ejection fraction in rats with acute myocardial infarction after six weeks of coronary artery occlusion [90]. What's more, probiotics can reduce myocardial cell apoptosis and improve ventricular remodelling in rats with spontaneous hypertension [91]. Saccharomyces boulardii can improve left atrial diameter and left ventricular ejection fraction in patients with chronic heart failure [92], and Lactobacillus Plantarum 299V can reduce infarct size and improve left ventricular function in rats [93]. However, probiotics remain at risk of probiotic translocation into the bloodstream and associated sepsis, and their safety needs additional study. Prebiotics is a dietary supplement, including isomalt oligosaccharides, oligosaccharides, Bifidus factors, and so on. It has a favourable effect on the host by selectively stimulating the growth and activity of bacteria. A recent study has shown that prebiotic oligofructose reduces infiltration of inflammatory cells in rats [94].

5.3. Fecal Microbiota Transplantation (FMT). FMT is a method of treating intestinal microecological imbalance and reconstructing normal intestinal function by introducing bacteria or metabolites from donor faeces into diseased receptors. At present, FMT is mostly used to treat Clostridium difficile infection. Most of the treatment cases have few side effects, but the application of FMT to other diseases is still unknown [95]. Many studies are examining the effectiveness of FMT in the treatment of chronic diseases and have found that there may be super-donor phenomena, that is to say, faeces from specific donors are more likely to make FMT successful than those from other donors [96]. Clinical studies have shown that autologous faecal transplantation can quickly restore the gut microbiota diversity of healthy people after the use of antibiotics [97]. In a randomised double-blind controlled trial involving 20 patients with metabolic syndrome, it was found that faecal flora of vegetarians after single transplantation could change the intestinal flora structure of some patients, but could not change the parameters related to vasculitis [98]. Also, when faecal bacteria are transplanted, viruses are transplanted [99]. Therefore, FMT has both advantages and disadvantages in the treatment of diseases. How to balance it is still a problem to solve. For patients with heart failure risk factors or existing heart failure, it is possible to reduce TMAO by transplanting low-yield TMAO gut microbiota, but there is no such clinical study.

5.4. Antibiotics. The antibiotic treatment destroys the balance of intestinal flora, leading to the decrease of flora abundance and changes in composition. Studies have shown that NSAIDs can alter intestinal flora composition in elderly patients and have adverse effects [100]. Other studies have shown that when antibiotics are injected to eliminate intestinal bacterial translocation, it can alleviate systemic inflammation and myocardial cell damage in mice with myocardial infarction [101]. In addition to bactericidal and bacteriostasis, rifaximin can also reduce the toxicity and translocation of bacteria, has an anti-inflammatory effect and positively regulates the composition of intestinal flora [102], and promotes the growth of bifidobacteria and lactobacillus [103]. Polymyxin B and Tobramycin can reduce the LPS in the intestine and faeces, and the contents of IL- $1 \beta$, IL- 6 and TNF- $\alpha$ in vivo in patients with heart failure [104]. However, improper use of antibiotics can kill beneficial bacteria in the body, making pathogens resistant and causing various adverse reactions. Therefore, we should weigh the side effects of antibiotics and their clinical effects.

\subsection{Microbial TMA-Lyase Inhibitors and Intestinal Mucosal}

Barrier Protectant. Some scholars have used choline analogues (compounds similar to choline) to inhibit the key enzyme CutC/D in the synthesis of TMA, thereby reducing the risk of cardiovascular disease by reducing the plasma TMAO level in mice [105]. Another recent study shows that resveratrol can stimulate the growth of beneficial bacteria in the intestinal 
tract through the reconstitution of intestinal microflora, thus decreasing the production of TMAO [103]. Urol is a metabolite of intestinal microflora derived from berries and pomegranate polyphenol. In vitro and mice, Urol and its synthetic analogue UAS03 can activate the pathways of aromatic hydrocarbon receptor (AhR) and nuclear factor red blood cell two related factor 2 (Nrf2) to enhance epithelial tight junction protein and enhance intestinal barrier function [106].

\section{Conclusion}

In conclusion, there are increasing shreds of evidence that gut microbiota disorders, intestinal barrier dysfunction and metabolites of gut microbiota are associated with heart failure. Intestinal barrier dysfunction and changes in the gut microbiota of composition may lead to abnormal production and absorption of gut microbiota metabolites in patients with heart failure. This imbalance can be expected to result in other complications such as heart dysfunction, inflammation, and so on. The composition of intestinal microflora in patients with heart failure is different from that in a healthy state. Reduction of SCFA-producing bacteria in patients with heart failure may be a noteworthy feature of patients with heart failure. In addition, the microbial potential for TMAO and LPS production increased significantly. More research has focused on the mechanism of microbial metabolites, and there is a need for clinical application of various therapeutic interventions. However, few studies have investigated in depth a direct role of the gut microbiota in heart failure and associated complications at the mechanistic and causal levels. Therefore, we need to further understand the role of gut microbiota in heart failure to better foster the development of diagnosis and treatment of heart failure.

\section{Conflicts of Interest}

The authors confirm that there are no conflicts of interest.

\section{Acknowledgments}

This work was supported by the Young Talent Lifting Project of China Association of Chinese Medicine (no. CACM-2018QNRC2-B04). This work was supported by the Ten thousand Talents Program of China (no. 20160621).

\section{References}

[1] J. J. McMurray, S. Adamopoulos, S. D. Anker et al., "ESC guidelines for the diagnosis and treatment of acute and chronic heart failure 2012: the task force for the diagnosis and treatment of acute and chronic heart failure 2012 of the European society of cardiology. Developed in collaboration with the heart failure association (HFA) of the ESC," European Heart Journal, vol. 33, no. 14, pp. 1787-1847, 2012.

[2] A. Mosterd and A. W. Hoes, "Clinical epidemiology of heart failure," Heart, vol. 93, no. 9, pp. 1137-1146, 2007.
[3] P. Ponikowski, A. A. Voors, S. D. Anker et al., "2016 ESC guidelines for the diagnosis and treatment of acute and chronic heart failure," European Heart Journal, vol. 37, no. 27, pp. 2129-2200, 2016.

[4] A. M. Shah and D. L. Mann, "In search of new therapeutic targets and strategies for heart failure: recent advances in basic science," The Lancet, vol. 378, no. 9792, pp. 704-712, 2011.

[5] F. Abi-Samra and D. Gutterman, "Cardiac contractility modulation: a novel approach for the treatment of heart failure," Heart Failure Reviews, vol. 21, no. 6, pp. 645-660, 2016.

[6] J. J. McMurray, M. Packer, A. S. Desai et al., "Angiotensinneprilysin inhibition versus enalapril in heart failure," New England Journal of Medicine, vol. 371, no. 11, pp. 993-1004, 2014.

[7] J. M. Brown and S. L. Hazen, "The gut microbial endocrine organ: bacterially derived signals driving cardiometabolic diseases," Annual Review of Medicine, vol. 66, no. 1, pp. 343-359, 2015.

[8] M. H. Floch, "Intestinal microecology in health and wellness," Journal of Clinical Gastroenterology, vol. 45, S108-10, 2011.

[9] L. K. Ursell, J. C. Clemente, J. R. Rideout, D. Gevers, J. G. Caporaso, and R. Knight, "The interpersonal and intrapersonal diversity of human-associated microbiota in key body sites," Journal of Allergy and Clinical Immunology, vol. 129, no. 5, pp. 1204-1208, 2012.

[10] R. Sender, S. Fuchs, and R. Milo, "Revised estimates for the number of human and bacteria cells in the body," PLoS Biology, vol. 14, no. 8, p. e1002533, 2016.

[11] L. Miele, V. Giorgio, M. A. Alberelli, E. De Candia, A. Gasbarrini, and A. Grieco, "Impact of gut microbiota on obesity, diabetes, and cardiovascular disease risk," Current Cardiology Reports, vol. 17, no. 12, 2015.

[12] R. N. Carmody, G. K. Gerber, J. M. LuevanoJr et al., "Diet dominates host genotype in shaping the murine gut microbiota," Cell Host \& Microbe, vol. 17, no. 1, pp. 72-84, 2015.

[13] S. Tamburini, N. Shen, H. C. Wu, and J. C. Clemente, "The microbiome in early life: implications for health outcome," Nature Medicine, vol. 22, no. 7, pp. 713-722, 2016.

[14] P. J. Turnbaugh, R. E. Ley, M. A. Mahowald, V. Magrini, E. R. Mardis, and J. I. Gordon, "An obesity-associated gut microbiome with increased capacity for energy harvest," Nature, vol. 444, no. 7122, pp. 1027-1031, 2006.

[15] F. Z. Marques, C. R. Mackay, and D. M. Kaye, "Beyond gut feelings: how the gut microbiota regulates blood pressure," Nature Reviews Cardiology, vol. 15, no. 1, pp. 20-32, 2018.

[16] W. H. Tang, T. Kitai, and S. L. Hazen, "Gut microbiota in cardiovascular health and disease," Circulation Research, vol. 120, no. 7, pp. 1183-1196, 2017.

[17] Z. Wang, E. Klipfell, B. J. Bennett et al., "Gut flora metabolism of phosphatidylcholine promotes cardiovascular disease," Nature, vol. 472, no. 7341, pp. 57-63, 2011.

[18] S. I. Sayin, A. Wahlstrom, J. Felin et al., "Gut microbiota regulates bile acid metabolism by reducing the levels of taurobeta-muricholic acid, a naturally occurring FXR antagonist," Cell Metabolism, vol. 17, no. 2, pp. 225-235, 2013.

[19] D. W. Russell, "The enzymes, regulation, and genetics of bile acid synthesis," Annual Review of Biochemistry, vol. 72, no. 1, pp. 137-174, 2003.

[20] M. K. Hamilton, G. Boudry, D. G. Lemay, and H. E. Raybould, "Changes in intestinal barrier function and gut microbiota 
in high-fat diet-fed rats are dynamic and region dependent," American Journal of Physiology-Gastrointestinal and Liver Physiology, vol. 308, no. 10, pp. G840-G851, 2015.

[21] R. Caesar, H. Nygren, M. Oresic, and F. Backhed, "Interaction between dietary lipids and gut microbiota regulates hepatic cholesterol metabolism," Journal of Lipid Research, vol. 57, no. 3, pp. 474-481, 2016.

[22] J. J. Bunker, T. M. Flynn, J. C. Koval et al., "Innate and adaptive humoral responses coat distinct commensal bacteria with immunoglobulin A," Immunity, vol. 43, no. 3, pp. 541-553, 2015.

[23] J. Li, F. Zhao, Y. Wang et al., "Gut microbiota dysbiosis contributes to the development of hypertension," Microbiome, vol. 5, no. 1, p. 14, 2017.

[24] Z. Wang, A. B. Roberts, J. A. Buffa et al., "Non-lethal inhibition of gut microbial trimethylamine production for the treatment of atherosclerosis," Cell, vol. 163, no. 7, pp. 1585-1595, 2015.

[25] X. Cui, L. Ye, J. Li et al., "Metagenomic and metabolomic analyses unveil dysbiosis of gut microbiota in chronic heart failure patients," Scientific Reports, vol. 8, no. 1, p. 635, 2018.

[26] E. Patterson, J. F. Cryan, G. F. Fitzgerald, R. P. Ross, T. G. Dinan, and C. Stanton, "Gut microbiota, the pharmabiotics they produce and host health," Proceedings of the Nutrition Society, vol. 73, no. 4, pp. 477-489, 2014.

[27] E. Pasini, R. Aquilani, C. Testa et al., "Pathogenic gut flora in patients with chronic heart failure," JACC: Heart Failure, vol. 4, no. 3, pp. 220-227, 2016.

[28] M. Luedde, T. Winkler, F. A. Heinsen et al., "Heart failure is associated with depletion of core intestinal microbiota," ESC Heart Failure, vol. 4, no. 3, pp. 282-290, 2017.

[29] T. Kamo, H. Akazawa, W. Suda et al., "Dysbiosis and compositional alterations with aging in the gut microbiota of patients with heart failure," PLoS One, vol. 12, no. 3, p. e0174099, 2017.

[30] M. Kummen, C. C. K. Mayerhofer, B. Vestad et al., "Gut microbiota signature in heart failure defined from profiling of 2 independent cohorts," Journal of the American College of Cardiology, vol. 71, no. 10, pp. 1184-1186, 2018.

[31] N. Arpaia, C. Campbell, X. Fan et al., "Metabolites produced by commensal bacteria promote peripheral regulatory $\mathrm{T}$-cell generation," Nature, vol. 504, no. 7480, pp. 451-455, 2013.

[32] Y. Nagatomo and W. H. Tang, "Intersections between microbiome and heart failure: revisiting the gut hypothesis," Journal of Cardiac Failure, vol. 21, no. 12, pp. 973-980, 2015.

[33] A. Sandek, J. Bauditz, A. Swidsinski et al., "Altered intestinal function in patients with chronic heart failure," Journal of the American College of Cardiology, vol. 50, no. 16, pp. 1561-1569, 2007.

[34] A. Sandek, A. Swidsinski, W. Schroedl et al., "Intestinal blood flow in patients with chronic heart failure," Journal of the American College of Cardiology, vol. 64, no. 11, pp. 1092-1102, 2014.

[35] A. Zabell and W. H. Tang, "Targeting the microbiome in heart failure," Current Treatment Options in Cardiovascular Medicine, vol. 19, no. 4, p. 27, 2017.

[36] T. Peschel, M. Schönauer, H. Thiele, S. Anker, G. Schuler, and J. Niebauer, "Invasive assessment of bacterial endotoxin and inflammatory cytokines in patients with acute heart failure," European Journal of Heart Failure, vol. 5, no. 5, pp. 609-614, 2003.

[37] E. Larsson, V. Tremaroli, Y. S. Lee et al., "Analysis of gut microbial regulation of host gene expression along the length of the gut and regulation of gut microbial ecology through MyD88," Gut, vol. 61, no. 8, pp. 1124-1131, 2012.

[38] A. L. Neves, J. Coelho, L. Couto, A. Leite-Moreira, and R. Roncon-Albuquerque, "Metabolic endotoxemia: a molecular link between obesity and cardiovascular risk," Journal of Molecular Endocrinology, vol. 51, no. 2, pp. R51-R64, 2013.

[39] S. D. Anker and S. von Haehling, "Inflammatory mediators in chronic heart failure: an overview," Heart, vol. 90, no. 4, pp. 464-470, 2004.

[40] Y. Y. Lam, C. W. Ha, C. R. Campbell et al., "Increased gut permeability and microbiota change associate with mesenteric fat inflammation and metabolic dysfunction in diet-induced obese mice," PLoS One, vol. 7, no. 3, p. e34233, 2012.

[41] P. D. Cani, R. Bibiloni, C. Knauf et al., "Changes in gut microbiota control metabolic endotoxemia-induced inflammation in high-fat diet-induced obesity and diabetes in mice," Diabetes, vol. 57, no. 6, pp. 1470-1481, 2008.

[42] A. Gomez, D. Luckey, C. J. Yeoman et al., "Loss of sex and age driven differences in the gut microbiome characterize arthritis-susceptible 0401 mice but not arthritis-resistant 0402 mice," PLoS One, vol. 7, no. 4, p. e36095, 2012.

[43] J. Yuan, A. L. Cao, M. Yu et al., "Th17 cells facilitate the humoral immune response in patients with acute viral myocarditis," Journal of Clinical Immunology, vol. 30, no. 2, pp. 226-234, 2010

[44] J. Yuan, M. Yu, Q. W. Lin et al., "Neutralization of IL-17 inhibits the production of anti-ANT autoantibodies in CVB3-induced acute viral myocarditis," International Immunopharmacology, vol. 10, no. 3, pp. 272-276, 2010.

[45] Y. H. Liao, N. Xia, S. F. Zhou et al., "Interleukin-17A contributes to myocardial ischemia/reperfusion injury by regulating cardiomyocyte apoptosis and neutrophil infiltration," Journal of the American College of Cardiology, vol. 59, no. 4, pp. 420-429, 2012.

[46] J. L. Round and S. K. Mazmanian, "Inducible Foxp3+ regulatory T-cell development by a commensal bacterium of the intestinal microbiota," Proceedings of the National Academy of Sciences, vol. 107, no. 27, pp. 12204-12209, 2010.

[47] M. Levy, A. A. Kolodziejczyk, C. A. Thaiss, and E. Elinav, "Dysbiosis and the immune system," Nature Reviews Immunology, vol. 17, no. 4, pp. 219-232, 2017.

[48] T. T. Tang, J. Yuan, Z. F. Zhu et al., "Regulatory T cells ameliorate cardiac remodeling after myocardial infarction," Basic Research in Cardiology, vol. 107, no. 1, 2012.

[49] M. Troseid, T. Ueland, J. R. Hov et al., "Microbiota-dependent metabolite trimethylamine- $\mathrm{N}$-oxide is associated with disease severity and survival of patients with chronic heart failure," Journal of Internal Medicine, vol. 277, no. 6, pp. 717-726, 2015.

[50] Y. Heianza, W. Ma, J. E. Manson, K. M. Rexrode, and L. Gut Qi, "Microbiota metabolites and risk of major adverse cardiovascular disease events and death: a systematic review and meta-analysis of prospective studies," Journal of the American Heart Association, vol. 6, no. 7, 2017.

[51] T. Suzuki, Y. Yazaki, A. A. Voors et al., "Association with outcomes and response to treatment of trimethylamine $\mathrm{N}$-oxide in heart failure (from BIOSTAT-CHF)," European Journal of Heart Failure, vol. 21, no. 7, pp. 877-886, 2018.

[52] W. H. Tang, Z. Wang, K. Shrestha et al., "Intestinal microbiotadependent phosphatidylcholine metabolites, diastolic dysfunction, and adverse clinical outcomes in chronic systolic 
heart failure," Journal of Cardiac Failure, vol. 21, no. 2, pp. 91-96, 2015.

[53] F. P. Martin, Y. Wang, N. Sprenger et al., "Probiotic modulation of symbiotic gut microbial-host metabolic interactions in a humanized microbiome mouse model," Molecular Systems Biology, vol. 4, no. 1, p. 157, 2008.

[54] W. Zhu, J. C. Gregory, E. Org et al., "Gut microbial metabolite TMAO enhances platelet hyperreactivity and thrombosis risk," Cell, vol. 165, no. 1, pp. 111-124, 2016.

[55] Z. Li, Z. Wu, J. Yan et al., "Gut microbe-derived metabolite trimethylamine N-oxide induces cardiac hypertrophy and fibrosis," Laboratory Investigation, vol. 99, no. 3, pp. 346-357, 2019.

[56] M. L. Chen, X. H. Zhu, L. Ran, H. D. Lang, L. Yi, and M. T. Mi, "Trimethylamine-N-Oxide induces vascular inflammation by activating the NLRP3 inflammasome through the SIRT3SOD2-mtROS signaling pathway," Journal of the American Heart Association, vol. 6, no. 9, 2017.

[57] M. M. Seldin, Y. Meng, H. Qi et al., "Trimethylamine N-oxide promotes vascular inflammation through signaling of mitogen-activated protein kinase and nuclear factor-kappaB," Journal of the American Heart Association, vol. 5, no. 2, 2016.

[58] G. Ma, B. Pan, Y. Chen et al., "Trimethylamine N-oxide in atherogenesis: impairing endothelial self-repair capacity and enhancing monocyte adhesion," Bioscience Reports, vol. 37, no. 2, p. BSR20160244, 2017.

[59] C. L. Organ, H. Otsuka, S. Bhushan et al., "Choline diet and its gut microbe-derived metabolite, trimethylamine $\mathrm{N}$-oxide, exacerbate pressure overload-induced heart failure," Circulation: Heart Failure, vol. 9, no. 1, p. e002314, 2016.

[60] W. H. Tang, Z. Wang, D. J. Kennedy et al., "Gut microbiotadependent trimethylamine $\mathrm{N}$-oxide (TMAO) pathway contributes to both development of renal insufficiency and mortality risk in chronic kidney disease," Circulation Research, vol. 116, no. 3, pp. 448-455, 2015.

[61] M. Savi, L. Bocchi, L. Bresciani et al., “Trimethylamine-NOxide (TMAO)-induced impairment of cardiomyocyte function and the protective role of Urolithin B-Glucuronide," Molecules, vol. 23, no. 3, 2018.

[62] B. J. Bennion and V. Daggett, "Counteraction of urea-induced protein denaturation by trimethylamine $\mathrm{N}$-oxide: a chemical chaperone at atomic resolution," Proceedings of the National Academy of Sciences, vol. 101, no. 17, pp. 6433-6438, 2004.

[63] S. D. Liberles and L. B. Buck, "A second class of chemosensory receptors in the olfactory epithelium," Nature, vol. 442, no. 7103, pp. 645-650, 2006.

[64] A. J. Brown, S. M. Goldsworthy, A. A. Barnes et al., “The orphan G protein-coupled receptors GPR41 and GPR43 are activated by propionate and other short chain carboxylic acids," Journal of Biological Chemistry, vol. 278, no. 13, pp. 11312-11319, 2003.

[65] A. Koh, F. De Vadder, P. Kovatcheva-Datchary, and F. Backhed, "From dietary fiber to host physiology: short-chain fatty acids as key bacterial metabolites," Cell, vol. 165, no. 6, pp. 1332-1345, 2016.

[66] J. Huang, L. Wang, S. Dahiya et al., "Histone/protein deacetylase 11 targeting promotes Foxp3+ Treg function," Scientific Reports, vol. 7, no. 1, 2017.

[67] C. J. Kelly, L. Zheng, E. L. Campbell et al., "Crosstalk between microbiota-derived short-chain fatty acids and intestinal epithelial HIF augments tissue barrier function," Cell Host \& Microbe, vol. 17, no. 5, pp. 662-671, 2015.

[68] T. W. H. Tang, H. C. Chen, C. Y. Chen et al., "Loss of abiota alters immune system composition and cripples postinfarction cardiac repair," Circulation, vol. 139, no. 5, pp. 647-659, 2019.

[69] C. C. K. Mayerhofer, T. Ueland, K. Broch et al., "Increased Secondary/primary bile acid ratio in chronic heart failure," Journal of Cardiac Failure, vol. 23, no. 9, pp. 666-671, 2017.

[70] W. H. W. Tang, D. Y. Li, and S. L. Hazen, "Dietary metabolism, the gut microbiome, and heart failure," Nature Reviews Cardiology, vol. 16, no. 3, pp. 137-154, 2019.

[71] Z. Eblimit, S. Thevananther, S. J. Karpen et al., "TGR5 activation induces cytoprotective changes in the heart and improves myocardial adaptability to physiologic, inotropic, and pressure-induced stress in mice," Cardiovascular Therapeutics, vol. 36, no. 5, p. e12462, 2018.

[72] A. Mancini, F. Campagna, P. Amodio, and K. M. Tuohy, "Gut : liver : brain axis: the microbial challenge in the hepatic encephalopathy," Food Function, vol. 9, no. 3, pp. 1373-1388, 2018.

[73] S. Bashiardes, H. Shapiro, S. Rozin, O. Shibolet, and E. Elinav, "Non-alcoholic fatty liver and the gut microbiota," Molecular Metabolism, vol. 5, no. 9, pp. 782-794, 2016.

[74] A. Xanthopoulos, R. C. Starling, T. Kitai, and F. Triposkiadis, "Heart failure and liver disease: cardiohepatic interactions," JACC: Heart Failure, vol. 7, no. 2, pp. 87-97, 2019.

[75] N. D. Vaziri, J. Wong, M. Pahl et al., "Chronic kidney disease alters intestinal microbial flora," Kidney International, vol. 83, no. 2, pp. 308-315, 2013.

[76] M. Kanbay, E. M. Onal, B. Afsar et al., "The crosstalk of gut microbiota and chronic kidney disease: role of inflammation, proteinuria, hypertension, and diabetes mellitus," International Urology and Nephrology, vol. 50, no. 8, pp. 1453-1466, 2018.

[77] C. Ronco, M. Haapio, A. A. House, N. Anavekar, and R. Bellomo, "Cardiorenal syndrome," Journal of the American College of Cardiology, vol. 52, no. 19, pp. 1527-1539, 2008.

[78] N. D. Vaziri, J. Yuan, S. Nazertehrani, Z. Ni, and S. Liu, "Chronic kidney disease causes disruption of gastric and small intestinal epithelial tight junction," American Journal of Nephrology, vol. 38, no. 2, pp. 99-103, 2013.

[79] F. Savira, L. Cao, I. Wang et al., "Apoptosis signal-regulating kinase 1 inhibition attenuates cardiac hypertrophy and cardiorenal fibrosis induced by uremic toxins: Implications for cardiorenal syndrome," PLoS One, vol. 12, no. 11, p. e0187459, 2017.

[80] M. V. S. Vanharanta, T. H. Rissanen, H. Adlercreutz, and J. T. Salonen, "Risk of cardiovascular disease-related and all-cause death according to serum concentrations of enterolactone," Archives of Internal Medicine, vol. 163, no. 9, pp. 1099-1104, 2003.

[81] R. Masella, C. Santangelo, M. D'Archivio, G. Li Volti, C. Giovannini, and F. Galvano, "Protocatechuic acid and human disease prevention: biological activities and molecular mechanisms," Current Medicinal Chemistry, vol. 19, no. 18, pp. 2901-2917, 2012.

[82] D. Wang, M. Xia, X. Yan et al., “Gut microbiota metabolism of anthocyanin promotes reverse cholesterol transport in mice via repressing miRNA-10b," Circulation Research, vol. 111, no. 8, pp. 967-981, 2012.

[83] L. A. David, C. F. Maurice, R. N. Carmody et al., "Diet rapidly and reproducibly alters the human gut microbiome," Nature, vol. 505, no. 7484, pp. 559-563, 2014. 
[84] A. Salehi-Abargouei, Z. Maghsoudi, F. Shirani, and L. Azadbakht, "Effects of dietary approaches to stop hypertension (DASH)-style diet on fatal or nonfatal cardiovascular diseases-incidence: a systematic review and meta-analysis on observational prospective studies," Nutrition, vol. 29, no. 4, pp. 611-618, 2013.

[85] E. B. Levitan, A. Wolk, and M. A. Mittleman, "Consistency with the DASH diet and incidence of heart failure," Archives of Internal Medicine, vol. 169, no. 9, pp. 851-857, 2009.

[86] L. Rifai, C. Pisano, J. Hayden, S. Sulo, and M. A. Silver, "Impact of the DASH diet on endothelial function, exercise capacity, and quality of life in patients with heart failure," Baylor University Medical Center Proceedings, vol. 28, no. 2, pp. 151-156, 2015.

[87] E. Lopez-Garcia, F. Rodriguez-Artalejo, T. Y. Li et al., “The mediterranean-style dietary pattern and mortality among men and women with cardiovascular disease," The American Journal of Clinical Nutrition, vol. 99, no. 1, pp. 172-180, 2014.

[88] F. De Filippis, N. Pellegrini, L. Vannini et al., "High-level adherence to a Mediterranean diet beneficially impacts the gut microbiota and associated metabolome," Gut, vol. 65, no. 11, pp. 1812-1821, 2016.

[89] F. Z. Marques, E. Nelson, P. Y. Chu et al., "High-fiber diet and acetate supplementation change the gut microbiota and prevent the development of hypertension and heart failure in hypertensive mice," Circulation, vol. 135, no. 10, pp. 964-977, 2017.

[90] X. T. Gan, G. Ettinger, C. X. Huang et al., "Probiotic administration attenuates myocardial hypertrophy and heart failure after myocardial infarction in the rat," Circulation: Heart Failure, vol. 7, no. 3, pp. 491-499, 2014.

[91] P. P. Lin, Y. M. Hsieh, W. W. Kuo et al., "Probiotic-fermented purple sweet potato yogurt activates compensatory IGF-IR/ PI3K/Akt survival pathways and attenuates cardiac apoptosis in the hearts of spontaneously hypertensive rats," International Journal of Molecular Medicine, vol. 32, no. 6, pp. 1319-1328, 2013.

[92] A. C. Costanza, S. D. Moscavitch, H. C. Faria Neto, and E. T. Mesquita, "Probiotic therapy with Saccharomyces boulardii for heart failure patients: a randomized, double-blind, placebocontrolled pilot trial," International Journal of Cardiology, vol. 179, pp. 348-350, 2015.

[93] V. Lam, J. Su, S. Koprowski et al., "Intestinal microbiota determine severity of myocardial infarction in rats," The FASEB Journal, vol. 26, no. 4, pp. 1727-1735, 2012.

[94] S. A. Kumar, L. C. Ward, and L. Brown, "Inulin oligofructose attenuates metabolic syndrome in high-carbohydrate, high-fat diet-fed rats," British Journal of Nutrition, vol. 116, no. 9, pp. 1502-1511, 2016.

[95] G. Cammarota, G. Ianiro, and A. Gasbarrini, "Fecal microbiota transplantation for the treatment of Clostridium difficile infection: a systematic review," Journal of Clinical Gastroenterology, vol. 48, no. 8, pp. 693-702, 2014.

[96] B. C. Wilson, T. Vatanen, W. S. Cutfield, and J. M. O'Sullivan, "The super-donor phenomenon in fecal microbiota transplantation," Frontiers in Cellular and Infection Microbiology, vol. 9, p. 2, 2019.

[97] Y. Taur, K. Coyte, J. Schluter et al., "Reconstitution of the gut microbiota of antibiotic-treated patients by autologous fecal microbiota transplant," Science Translational Medicine, vol. 10, no. 460, p. eaap9489, 2018.
[98] L. P. Smits, R. S. Kootte, E. Levin et al., "Effect of vegan fecal microbiota transplantation on carnitine- and cholinederived trimethylamine-N-oxide production and vascular inflammation in patients with metabolic syndrome," Journal of the American Heart Association, vol. 7, no. 7, 2018.

[99] C. Chehoud, A. Dryga, Y. Hwang et al., "Transfer of viral communities between human individuals during fecal microbiota transplantation," mBio, vol. 7, no. 2, 2016.

[100] K. Tiihonen, S. Tynkkynen, A. Ouwehand, T. Ahlroos, and N. Rautonen, "The effect of ageing with and without non-steroidal anti-inflammatory drugs on gastrointestinal microbiology and immunology," British Journal of Nutrition, vol. 100, no. 1, pp. 130-137, 2008.

[101] X. Zhou, J. Li, J. Guo et al., "Gut-dependent microbial translocation induces inflammation and cardiovascular events after ST-elevation myocardial infarction," Microbiome, vol. 6, no. $1,2018$.

[102] F. R. Ponziani, M. A. Zocco, F. D'Aversa, M. Pompili, and A. Gasbarrini, "Eubiotic properties of rifaximin: disruption of the traditional concepts in gut microbiota modulation," World Journal of Gastroenterology, vol. 23, no. 25, pp. 4491-4499, 2017.

[103] M. L. Chen, L. Yi, Y. Zhang et al., "Resveratrol attenuates Trimethylamine-N-Oxide (TMAO)-induced atherosclerosis by regulating TMAO synthesis and bile acid metabolism via remodeling of the gut microbiota," $m B i o$, vol. 7 , no. 2 , pp. e02210-02215, 2016.

[104] V. M. Conraads, P. G. Jorens, L. S. De Clerck et al., "Selective intestinal decontamination in advanced chronic heart failure: a pilot trial," European Journal of Heart Failure, vol. 6, no. 4, pp. 483-491, 2004.

[105] A. B. Roberts, X. Gu, J. A. Buffa et al., "Development of a gut microbe-targeted nonlethal therapeutic to inhibit thrombosis potential," Nature Medicine, vol. 24, no. 9, pp. 1407-1417, 2018.

[106] R. Singh, S. Chandrashekharappa, S. R. Bodduluri et al., "Enhancement of the gut barrier integrity by a microbial metabolite through the Nrf2 pathway," Nature Communications, vol. 10, no. 1, p. 89, 2019. 


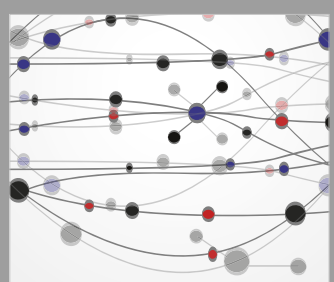

The Scientific World Journal
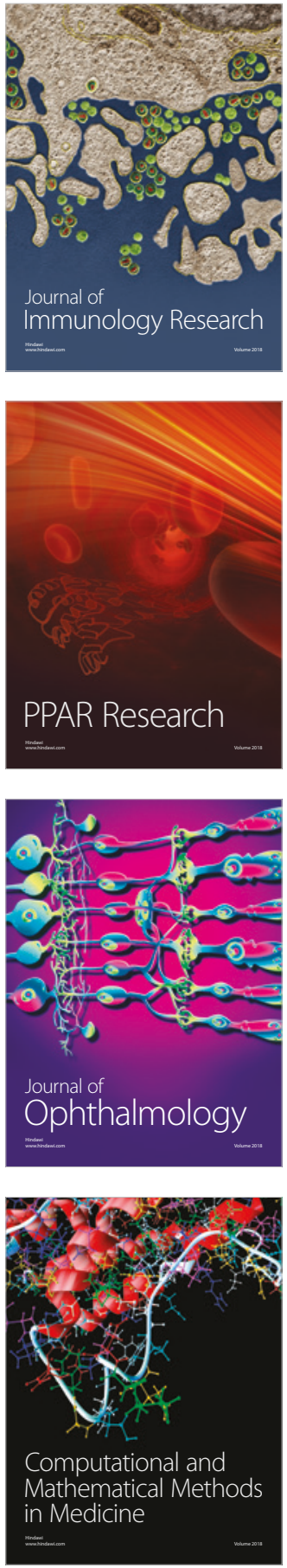

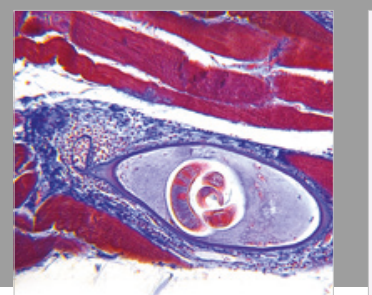

Gastroenterology Research and Practice

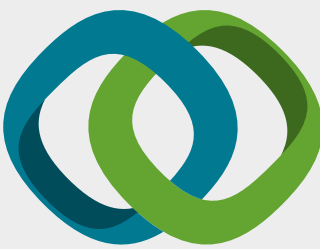

\section{Hindawi}

Submit your manuscripts at

www.hindawi.com
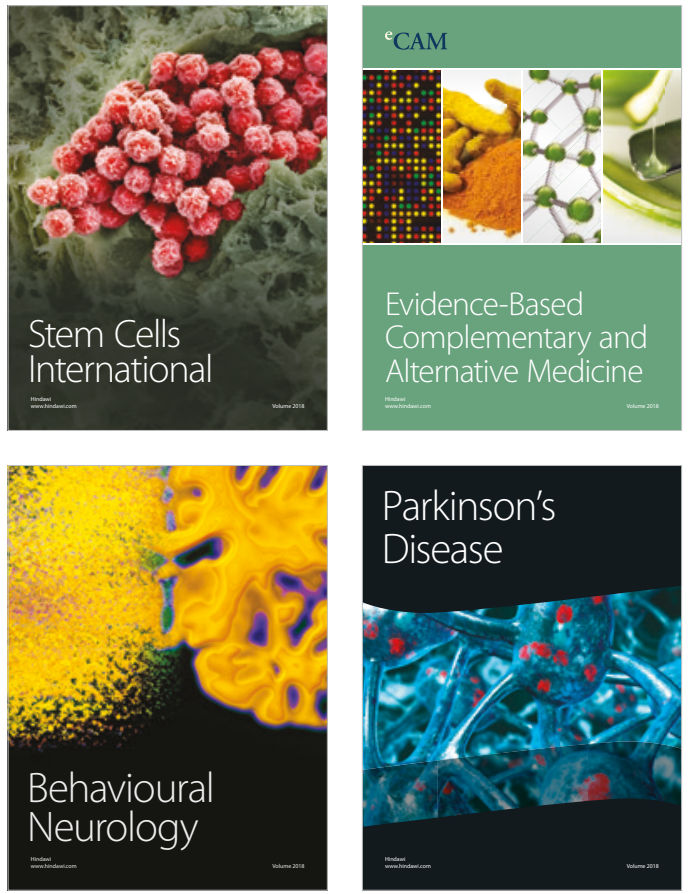

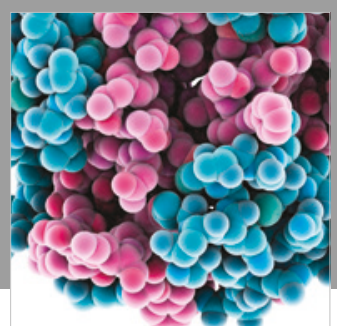

ournal of

Diabetes Research

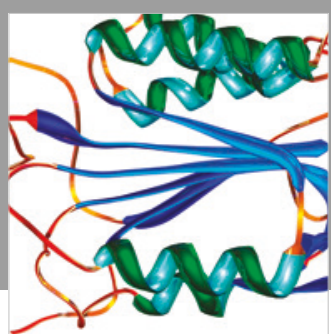

Disease Markers
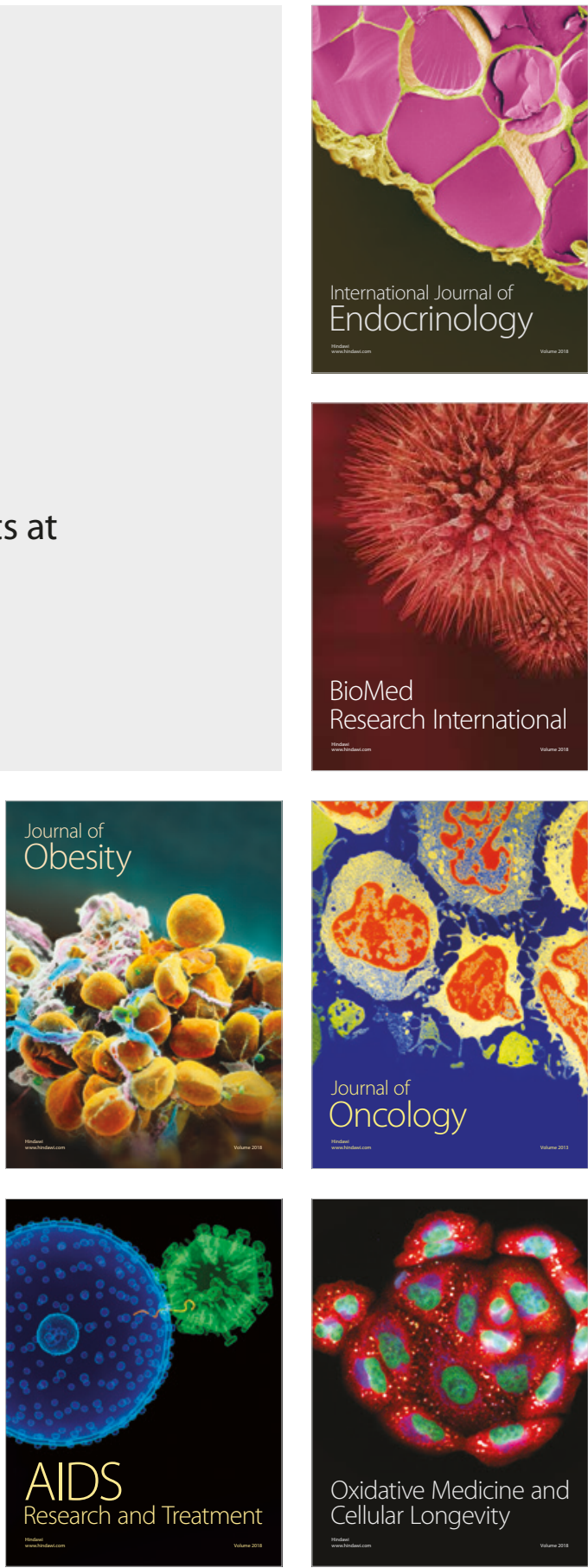